\title{
Diabetes, hyperglycaemia and mortality in disabled older women: The Women's Health and Ageing Study I
}

\author{
C. S. Blaum*†, S. Volpato§, A. R. Cappolaף, P. Chaves ${ }^{* *}$, Q. L. Xue†‡, J. M. Guralnik†† and \\ L. P. Fried†‡
}

*Department of Medicine, The University of Michigan, Ann Arbor, Ml and Departments of tMedicine and $\ddagger$ Epidemiology, The Johns Hopkins Medical Institutions, Baltimore, MD, USA, §Dipartimento di Medicina, Universita di Ferrara, Ferrara, Italy, qDepartment of Medicine, University of Maryland, Baltimore, MD, USA, ${ }^{* *}$ Centre for Studies on Ageing and Care for the Elderly (UNATI), Rio de Janeiro State University (UERJ), Rio de Janeiro, Brazil, and +tLaboratory of Epidemiology, Demography, and Biometry, National Institute on Aging, Bethesda, MD, USA

Accepted 1 June 2004

\begin{abstract}
Aims Diabetes is associated with increased mortality in older adults, but the specific contributions of diabetes-associated clinical conditions and of increasing hyperglycaemia to mortality risk are unknown. We evaluated whether cardiovascular disease, comorbidities, or degree of hyperglycaemia, particularly severe hyperglycaemia, affected diabetes-related mortality risk in older, disabled women.

Methods Six-year mortality follow-up of a random sample of 576 disabled women (aged 65-101 years), recruited from the Medicare eligibility list in Baltimore (MD, USA). All-cause and cardiovascular mortality were evaluated by diabetes status: no diabetes; diabetes with mild, moderate, and severe hyperglycaemia [defined by tertiles of glycosylated haemoglobin (GHB) among women with diabetes].
\end{abstract}

Results Diabetes with mild, moderate, and severe hyperglycaemia was associated with an increased hazard rate (HR) for all-cause mortality, even after adjustment for demographics, risks for cardiovascular disease, cardiovascular and noncardiovascular conditions, and other known mortality risks. A dose-response effect was suggested [mild hyperglycaemia, HR 1.81, 95\% confidence interval (CI) 1.03, 3.17; moderate hyperglycaemia, HR 2.02, $95 \%$ CI 1.34, 3.57; severe hyperglycaemia, HR $2.22,95 \%$ CI $1.17,4.25]$. Women with diabetes had a significantly increased HR for non-cardiovascular death, but not for cardiovascular death, compared with those without diabetes.

Conclusions Diabetes, whether characterized by mild, moderate or severe hyperglycaemia, appears to be an independent risk factor for excess mortality in older disabled women and this risk may increase with increasing hyperglycaemia. This mortality risk is not completely explained by vascular complications, and involves non-cardiovascular deaths. Risks and benefits of diabetes management, including glycaemic control and management of vascular and other comorbidities, should be studied in older people with complications and comorbidities.

Diabet. Med. 22, 543-550 (2005)

Keywords diabetes, hyperglycaemia, mortality
Correspondence to: Caroline S. Blaum MD, Assistant Professor of Internal Medicine, Division of Geriatrics, The University of Michigan Medical Center, Room 1111-CCGCB, 1500 East Medical Center Drive, Ann Arbor, Ml 481090926, USA. E-mail: cblaum@umich.edu

\section{Introduction}

Type 2 diabetes mellitus has been associated with increased mortality among both middle-aged and older people in population-based studies [1-13]. Most researchers have found an increased mortality risk for older adults with diabetes compared with those without diabetes, although of decreased 
magnitude than the diabetes-associated mortality among younger adults. This excess mortality is found particularly for cardiovascular causes of death, but also for all-cause and noncardiovascular death $[1,3,6,8,10-13]$. It remains unclear if the effect of diabetes on mortality is still present when multiple comorbidities and competing mortality risks, particularly cardiovascular conditions, are taken into account, or if an effect of diabetes on mortality is related to level of hyperglycaemia.

Older adults with multiple chronic conditions and functional limitations represent a rapidly growing population with a high prevalence of diabetes. Management of diabetes, particularly of hyperglycaemia, remains controversial in this group. Their morbidity and mortality may be more attributable to accumulated effects of hypertension and cardiovascular diseases; any independent effect of diabetes on mortality might be insignificant due to competing comorbidities related or unrelated to diabetes. No studies have evaluated a population of older adults with extensive comorbidities and disabilities to determine if diabetes continues to be associated with excess mortality after accounting for vascular diseases and other competing mortality risks.

Nor is it known whether diabetes-related mortality is related to level of hyperglycaemia in older people with complications and comorbidities. Recently, Khaw and colleagues reported that in a cohort of European men aged 45-79 years, increasing glycated haemoglobin explained much of the excess risk of mortality due to diabetes [14]. If severe hyperglycaemia were responsible for the independent effect of diabetes on mortality in older disabled women, that would suggest a continuing adverse effect of hyperglycaemia even in the presence of diabetes complications and comorbidities, raising the question of whether hyperglycaemia management would affect mortality risk.

This study used data from a population-based prospective study of moderately and severely disabled women living in the community, The Women's Health and Ageing Study I (WHAS I) [15] to test two hypotheses: (i) that older disabled women with diabetes have an increased risk of mortality compared with those without diabetes, even after cardiovascular risks, comorbidities, and other mortality risks are taken into account; and (ii) that this independent risk is found in women with severe hyperglycaemia rather than those with mild or moderate hyperglycaemia or normoglycaemia. In addition, because vascular disease is thought to be a leading cause of the increased mortality risk in diabetes, we compared risk for cardiovascular and non-cardiovascular mortality in older disabled women with and without diabetes.

\section{Methods}

Data were from the baseline and mortality surveillance followup data of the WHAS I. WHAS I is a prospective study of 1002 community-dwelling women in the Baltimore area aged $\geq 65$ years who were moderately to severely disabled at baseline. The study was conducted by the Johns Hopkins Medical
Institutions and was sponsored by the Epidemiology, Demography and Biometry Program of the National Institute on Aging. Subject sampling and recruitment has been described [15]. An age-stratified random sample of women $\geq 65$ years was screened to identify the $1 / 3$ most disabled women living in the community. Eligibility was determined by self-reported difficulty in tasks in two or more domains of functioning (of four domains [16]) and no significant dementia [Mini-Mental State Examination (MMSE) score of 18 or greater] [17]. Standardized baseline evaluations, interviews and physical examinations were done in the homes of the participants (instruments used and data collected have been published [15,18]). Blood samples were obtained from 784 subjects, processed, placed on ice and sent the same day to the Quest Diagnostic Laboratories in Teterboro (NJ, USA). Because our research concerned a measure of glycosylated haemoglobin (GHB), our sample was restricted to those women for whom baseline bloods were available within 90 days of the baseline evaluation, and the same laboratory technique was used (576, see details below). Participants who did not provide blood samples were older and had more disability in activities of daily living but were not different in body mass index (BMI) or number of chronic diseases. The study was approved by the Institutional Review Board of the Johns Hopkins Medical Institutions and all participants gave informed consent.

\section{Variables and their measurement}

\section{Diabetes and comorbidities}

Diabetes was ascertained at baseline using a specific standardized algorithm [19] that utilized data from multiple sources. Sources included the baseline interview, a standardized nurse's examination in the home, and medications based on review of medication bottles, non-fasting blood test results, medical records, and questionnaires sent to the participant's primary care physicians, and have been previously described [20]. Four baseline diabetes status variables were defined: (i) no diabetes ( $n=465)$; (ii) diabetes with mild hyperglycaemia, lowest tertile (approximate) of elevated GHB among women with diabetes $[\mathrm{GHB}<9.6, n=41$ (see below for details of GHB determination)]; (iii) diabetes with moderate hyperglycaemia, middle tertile (GHB between 9.6 and 11, n= 40); and (iv) diabetes with severe hyperglycaemia, $\mathrm{GHB} \geq 11 \%$ (top tertile of $\mathrm{GHB}$, $n=33$ ). (Note that the GHB test, as discussed in detail below, is not the same as $\mathrm{HbA}_{1 \mathrm{c}}$ used currently. Also, the tertiles are approximate due to similar GHB values among some women.)

Disease-specific algorithms using data from the multiple sources noted above were also used to evaluate the effect of diseases associated with mortality: both related to diabetes and atherosclerosis [congestive heart failure (CHF), myocardial infarction (MI), angina, stroke] or unrelated (respiratory conditions, cancer). Peripheral arterial disease (PAD) was assessed by the ankle-brachial index, measured using a Doppler stethoscope (Parks Model 841-A). An ankle-brachial index value $<0.9$ was considered diagnostic [21].

\section{Other baseline covariates}

Other variables related to diabetes complications, mortality and mortality risk were obtained at baseline evaluation and used as covariates. Demographic variables included age and 
race (African-American vs. not). Socioeconomic status was measured by educational attainment ( 8 th grade vs. greater) and income (lowest tertile). Variables associated with risk of mortality included: BMI, measured during baseline physical evaluation and categorized into three levels (overweight $=$ BMI 85 th percentile or above, thin = BMI 15 th percentile or below vs. all others [20,22]), smoking (currently smoking/quit within the last year vs. other); cholesterol level (only non-fasting was available; this was categorized into high $>6.2$ and low < 4.9) [23] and hypertension. Depressive symptoms (14 depressive answers out of 30 on the Geriatric Depression Scale [24,25]) and cognitive performance in the lowest quartile (score of 18-25 on baseline Folstein MMSE) were also evaluated.

\section{Glycosylated haemoglobin determination}

Non-fasting glycohaemoglobin (GHB) was measured using low-pressure cation exchange chromatography (reference range 5-8.6\%; 765 Glycomat, Palo Alto, CA, USA). This particular measure is available for 576 of the 631 women with baseline blood tests because the laboratory technique was changed as baseline evaluations were being completed. Our final study sample is therefore restricted to these 576 women. Although this 1993 glycosylated haemoglobin measurement cannot be directly referenced to measures used in the DCCT [26], the reference values for this test are available (5-8.6\%). Of women with adjudicated diabetes, values ranged from 8 to $20 \%$; women with diabetes were categorized as having mild, moderate or severe hyperglycaemia based on GHB level as described above.

\section{Mortality follow-up}

Vital status has been determined through 1999 using follow-up interviews with proxies, obituaries, and matching to the $\mathrm{Na}$ tional Death Index (women who died among our study sample $=177$ ). For all women, this was between 5 and 6 years after study entry. Death certificates have been obtained for all but eight of these decedents. Cause-specific mortality was based on the underlying cause of death coded according to the International Classification of Diseases as any cardiovascular mortality (codes 390-459; 81 deaths) or all other mortality (88 deaths) [27].

\section{Statistical analysis}

Standard descriptive techniques were used to study the demographic and health-related characteristics of the study sample by baseline diabetes status (i.e. no diabetes, diabetes with mild, moderate and severe hyperglycaemia). Differences among these groups were evaluated for significance by ANOVA (for continuous variables) and Wilcoxon rank sum test for trend (for categorical variables) [28].

Kaplan-Meier analysis was used to study the age-adjusted survival function of the sample. In this analysis, the mild and moderate groups were combined and the analysis compared survival in those without diabetes with two other groups: a combined group with mild/moderate hyperglycaemia, and the group with severe hyperglycaemia. This was done to test specifically the impact of severe hyperglycaemia on survival.

Cox proportional hazard regression models were then used to evaluate the HR for mortality according to baseline diabetes status, adjusting for covariates described above. These models estimated the effects of baseline diabetes status as measured by four analytic groups (i.e. no diabetes, diabetes with mild, moderate or severe hyperglycaemia) in order to investigate further the role of mild and moderate, as well as severe hyperglycaemia. Adjustment for confounding covariates on mortality was done by adding sequentially entered blocks of variables to the regression model: (i) demographic risk factors; (ii) demographic factors plus variables potentially related to general mortality risk (smoking, high and low cholesterol levels, high and low BMI, hypertension); (iii) the measures in (ii) plus adjudicated comorbid diseases in the pathway from diabetes to mortality (stroke, cardiac disease, PAD); the measures in (iii) plus competing causes of death (respiratory disease, cancer) and confounders (lower cognitive score, depressive symptoms). The proportional hazard assumption was tested by creating a time-dependent binary 'phase' variable indicating different follow-up periods $(0$ $=0$ through $\leq 3$ years; $1=\geq 3$ through 6 years). Then we added a phase main effect variable and variables for the interaction between the phase and baseline diabetes/hyperglycaemia status to the fully adjusted model.

To evaluate the HRs for cardiovascular and non-cardiovascular mortality, the mild/moderate groups were again combined due to the small number of deaths. Separate models were run for each of these two causes of death, treating cases with the other cause as censored cases. All models are adjusted for the covariates described in the preceding paragraph.

The statistical programs used were SAS Version 8 (SAS Institute Inc., Cary, NC, USA) [29] for data management and Stata Version 7 (StataCorp LP, College Station, TX, USA) [30] for data analysis.

\section{Results}

Table 1 shows the baseline demographic and health-related characteristics of the study participants by diabetes status. Across the four groups, from no diabetes, to diabetes with mild or moderate hyperglycaemia, to diabetes with severe hyperglycaemia, there were significant trends of decreasing age, increasing BMI, and increasing GHB. Among women with diabetes, there was a significant trend of increasing oral hypoglycaemic medication use, and women with moderate and severe hypoglycaemia were more likely to use insulin. Those with severe hyperglycaemia reported longer diabetes duration than the other two groups.

There was no significant difference in the prevalences of most chronic diseases by glycohaemoglobin level among those with diabetes. In general, one or several of the diabetes groups had higher prevalences of hypertension, MI, stroke, PAD and depressive symptoms than those without diabetes, but there were no significant trends in this sample. Respiratory diseases, cancer and lower MMSE scores showed no significant trend in prevalence among the groups.

The age-adjusted survival for three analytical groups is displayed in the Kaplan-Meier survival curve in Fig. 1. Diabetes groups with mild and moderate hyperglycaemia were combined in this analysis. Diabetes with mild/moderate hyperglycaemia 
Table 1 Baseline demographic and health characteristics by diabetes status

\begin{tabular}{|c|c|c|c|c|}
\hline Characteristic & $\begin{array}{l}\text { No diabetes } \\
(n=463)\end{array}$ & $\begin{array}{l}\text { Diabetes, mild } \\
\text { hyperglycaemia } \\
(n=41)\end{array}$ & $\begin{array}{l}\text { Diabetes, moderate } \\
\text { hyperglycaemia } \\
(n=40)\end{array}$ & $\begin{array}{l}\text { Diabetes, severe } \\
\text { hyperglycaemia } \\
(n=32)\end{array}$ \\
\hline Age (years) $(\text { mean } \pm s D)^{*}$ & $78.2 \pm 7.9$ & $75.6 \pm 7.1$ & $74.5 \pm 6.3$ & $73.1 \pm 5.6$ \\
\hline African-American (\%) & 21 & 46 & 30 & 50 \\
\hline Education $<9$ years $(\%)$ & 43 & 41 & 53 & 34 \\
\hline Low income $(\%)$ & 30 & 29 & 35 & 28 \\
\hline Body mass index, low $(\%)^{*}$ & 12 & 7 & 0 & 0 \\
\hline Body mass index, high $(\%)^{*}$ & 22 & 29 & 38 & 47 \\
\hline Body mass index (mean $\pm S D)^{*}$ & $28.0 \pm 6.6$ & $29.6 \pm 6.9$ & $32.5 \pm 6.3$ & $33.5 \pm 7.1$ \\
\hline Oral hypoglycaemics (\%)† & & 44 & 53 & 63 \\
\hline Insulin $(\%)$ & & 10 & 33 & 25 \\
\hline Duration diabetes (years) (mean \pm SD) & & $15.9 \pm 14.8$ & $13.2 \pm 9.9$ & $19.3 \pm 17.2$ \\
\hline Mean glycosylated haemoglobin $( \pm S D)^{*}$ & $7.6 \pm 0.9$ & $7.9 \pm 0.6$ & $9.8 \pm 0.6$ & $12.5 \pm 1.4$ \\
\hline Current smoker or recently stopped (\%) & 16 & 17 & 8 & 12 \\
\hline Total cholesterol (mean mmol/1 \pm sD) & $5.8 \pm 1.1$ & $5.6 \pm 1.1$ & $6.6 \pm 1.2$ & $6.0 \pm 1.0$ \\
\hline Cholesterol > $6.2 \mathrm{mmol} / 1(\%)$ & 32 & 22 & 33 & 44 \\
\hline Cholesterol < $4.9 \mathrm{mmol} / 1(\%)$ & 19 & 20 & 23 & 16 \\
\hline Hypertension $(\%)^{*}$ & 53 & 73 & 68 & 91 \\
\hline Angina (\%) & 28 & 34 & 48 & 41 \\
\hline Myocardial infarction (\%) & 15 & 15 & 18 & 22 \\
\hline Congestive heart failure ( $\%$ ) & 24 & 32 & 33 & 28 \\
\hline Stroke $(\%)$ & 10 & 12 & 23 & 19 \\
\hline Peripheral arterial disease $(\%)$ & 27 & 39 & 50 & 44 \\
\hline Cancer $(\%)$ & 12 & 32 & 15 & 12 \\
\hline Respiratory (\%) & 44 & 59 & 50 & 50 \\
\hline Depressive symptoms (\%) & 16 & 12 & 35 & 31 \\
\hline MMSE score 18-25 (\%) & 30 & 37 & 30 & 22 \\
\hline
\end{tabular}

MMSE, Mini-Mental State Examination.

$* P<0.01$ for trend across the four groups.

$\dagger P<0.01$ for trend across the three groups.

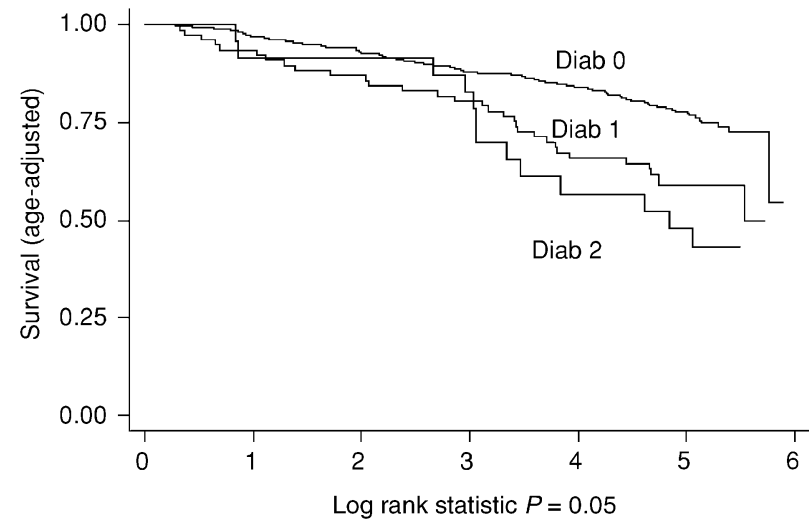

Figure 1 The Kaplain-Meier survival curve for the three analytic groups of disabled women-those without diabetes, those with diabetes and mild to moderate hyperglycemia [glycosylated haemoglobin (GHB) $<11 \%$ measured using low-pressure cation exchange chromatography, Ciba Corning 765 Glycomat; reference range 5-8.6\%], and those with severe hyperglycemia $(\mathrm{GHB} \geq 11 \%)$. Vital status was ascertained between 5 and 6 years from study entry for all the women. Diab $0=$ no diabetes (normoglycaemia); Diab $1=$ diabetes with mild or moderate hyperglycaemia; Diab 2, diabetes with severe hyperglycaemia. and particularly diabetes with severe hyperglycaemia were all associated with decreased survival, compared with no diabetes. There is a gradient of decreased survival across the three groups, from best survival for the no-diabetes group to worst survival for the severe hyperglycaemia group. The log rank statistic was $P=0.05$, indicating a borderline statistically significant difference among the curves. Formal testing for the presence of a non-proportional hazard, indicating a potential interaction between diabetes status and follow-up time, was not significant.

Table 2 presents results from the sequential Cox proportional hazards regression models assessing the HR for all-cause mortality for women with no diabetes, and diabetes with mild, moderate and severe hyperglycaemia, adjusting for key covariates. The reference group is the group without diabetes and this analysis displays explicitly the trend of increasing mortality with increasing level of hyperglycaemia. The models build from the baseline model, adjusted only for age, to a fully adjusted model by sequentially adjusting for different categories of covariates. In the fully adjusted model, among all the covariates tested, diabetes with severe hyperglycaemia had the largest HR, and diabetes with moderate hyperglycaemia had the second largest HR, for all-cause mortality. The test for trend of increasing HR by group (normoglycaemia, mild, 
Table 2 Crude and adjusted hazard rate (HR) of diabetes with mild, moderate and severe hyperglycaemia on mortality in moderately to severely disabled older women

\begin{tabular}{|c|c|c|c|c|c|}
\hline Characteristic & $\begin{array}{l}\text { Baseline model } \\
\text { (age-adjusted) }\end{array}$ & Model 1 & Model 2 & Model 3 & Model 4 \\
\hline Diabetes—mild hyperglycaemia & $1.67(1.00,2.82)$ & $1.68(1.00,2.84)$ & $1.67(1.00,2.83)$ & $1.75(1.03,3.00)$ & $1.81(1.03,3.17)$ \\
\hline Diabetes-moderate hyperglycaemia & $2.14(1.26,3.64)$ & $2.08(1.22,3.53)$ & $2.51(1.45,4.34)$ & $1.96(1.11,3.48)$ & $2.02(1.14,3.57)$ \\
\hline Diabetes—severe hyperglycaemia & $2.56(1.42,4.63)$ & $2.54(1.40,4.62)$ & $2.97(1.61,5.48)$ & $2.30(1.23,4.32)$ & $2.23(1.17,4.22)$ \\
\hline Age (1 year) & $1.08(1.06,1.10)$ & $1.08(1.05,1.10)$ & $1.07(1.05,1.10)$ & $1.07(1.04,1.10)$ & $1.07(1.04,1.09)$ \\
\hline Black & & $0.92(0.63,1.32)$ & $0.82(0.57,1.20)$ & $0.79(0.54,1.16)$ & $0.80(0.54,1.20)$ \\
\hline Low education & & $1.16(0.85,1.57)$ & $1.16(0.84,1.59)$ & $1.11(0.81,1.53)$ & $1.08(0.78,1.51)$ \\
\hline Low income & & $1.22(0.88,1.69)$ & $1.20(0.86,1.66)$ & $1.09(0.78,1.52)$ & $1.06(0.75,1.49)$ \\
\hline Current/recent smoker & & & $1.57(1.03,2.40)$ & $1.48(0.95,2.23)$ & $1.41(0.90,2.21)$ \\
\hline Body mass index-low & & & $1.89(1.24,2.88)$ & $1.93(1.26,2.94)$ & $1.92(1.25,2.93)$ \\
\hline Body mass index-high & & & $0.86(0.57,1.31)$ & $0.95(0.62,1.45)$ & $0.89(0.56,1.38)$ \\
\hline Cholesterol—high & & & $1.10(0.77,1.59)$ & $1.14(0.79,1.64)$ & $1.17(0.82,1.70)$ \\
\hline Cholesterol-low & & & $1.77(1.23,2.58)$ & $1.90(1.31,2.76)$ & $1.86(1.28,2.72)$ \\
\hline Hypertension & & & $1.18(0.86,1.62)$ & $1.11(0.81,1.52)$ & $1.11(0.80,1.53)$ \\
\hline Congestive heart failure & & & & $1.66(1.19,2.31)$ & $1.60(1.14,2.25)$ \\
\hline Stroke & & & & $1.54(1.02,2.33)$ & $1.54(1.00,2.37)$ \\
\hline Myocardial infarction & & & & $1.67(1.16,2.41)$ & $1.66(1.14,2.42)$ \\
\hline Peripheral arterial disease & & & & $1.61(1.16,2.22)$ & $1.55(1.12,2.16)$ \\
\hline Angina & & & & $0.89(0.64,1.25)$ & $0.94(0.66,1.32)$ \\
\hline Cancer & & & & & $0.93(0.57,1.49)$ \\
\hline Respiratory & & & & & $1.08(0.78,1.50)$ \\
\hline MMSE score 18-25 & & & & & $1.22(0.87,1.70)$ \\
\hline Depressive symptoms & & & & & $1.22(0.83,1.79)$ \\
\hline
\end{tabular}

MMSE, Mini-Mental State Examination.

Baseline model—age-adjusted only. Reference group for all models = no-diabetes group

Model 1, adjusted for age, African-American, education, low income. Model 2, model 1 plus risks (smoker, body mass index, cholesterol, hypertension). Model 3, model 2 plus cardiovascular diseases (adjudicated). Model 4, model 3 plus non-cardiovascular conditions (cancer, respiratory conditions, MMS score 18-25, depressive syptoms).

Table 3 Association between baseline diabetes status and cause-specific mortality in older disabled women: 6 years' follow-up

\begin{tabular}{|c|c|c|c|c|c|c|}
\hline & \multicolumn{3}{|c|}{ Cardiovascular mortality: HR (RR) and $95 \% \mathrm{CI}$} & \multicolumn{3}{|c|}{ Non-cardiovascular mortality: RR and 95\% CI } \\
\hline & $\begin{array}{l}\text { No. of } \\
\text { deaths }\end{array}$ & $\begin{array}{l}\text { Age-adjusted } \\
\text { RR }\end{array}$ & $\begin{array}{l}\text { Fully adjusted } \\
\mathrm{RR}^{*}\end{array}$ & $\begin{array}{l}\text { No. of } \\
\text { deaths }\end{array}$ & $\begin{array}{l}\text { Age-adjusted } \\
\text { RR }\end{array}$ & $\begin{array}{l}\text { Fully adjusted } \\
\mathrm{RR}^{*}\end{array}$ \\
\hline No diabetes & 66 & 1 & 1 & 61 & 1 & 1 \\
\hline $\begin{array}{l}\text { Diabetes_mild/moderate } \\
\text { hyperglycaemia }\end{array}$ & 12 & $\begin{array}{l}1.46 \\
(0.78,2.72)\end{array}$ & $\begin{array}{l}1.70 \\
(0.84,3.39)\end{array}$ & 20 & $\begin{array}{l}2.42 \\
(1.45,4.05)\end{array}$ & $\begin{array}{l}2.25 \\
(1.28,3.95)\end{array}$ \\
\hline Diabetes—severe hyperglycaemia & 3 & $\begin{array}{l}1.25 \\
(0.39,4.07)\end{array}$ & $\begin{array}{l}1.02 \\
(0.29,3.54)\end{array}$ & 7 & $\begin{array}{l}2.75 \\
(1.22,6.16)\end{array}$ & $\begin{array}{l}2.47 \\
(1.01,6.06)\end{array}$ \\
\hline
\end{tabular}

"Adjusted for age, race, low educational attainment, low income, smoking, body mass index (high and low), cholesterol levels (high and low), hypertension, angina, myocardial infarction, congestive heart failure, stroke, peripheral vascular disease, cancer, respiratory conditions, MMSE score 18-25, depressive symptoms.

(Eight deaths are missing because cause was not available.)

MMSE, Mini-Mental State Examination.

moderate and severe hyperglycaemia) was highly significant $(P<0.001)$. Other variables with a significant effect on allcause mortality included smoking, low BMI, low cholesterol, CHF, MI, stroke and PAD.

Table 3 compares the associations of baseline diabetes status with cardiovascular and non-cardiovascular mortality over the 6 years of follow-up. The number of deaths for the diabetes groups is small so the mild and moderate group were again combined. The HR for cardiovascular deaths did not achieve statistical significance for either group (mild/moderate, and severe) compared with the group without diabetes. However, the HRs for non-cardiovascular deaths, both age-adjusted and fully adjusted, were large and statistically significant for women with diabetes in both groups, relative to the group without diabetes. 


\section{Discussion}

Our results demonstrate that in older women with moderate to severe disability, and numerous other potential causes of mortality, those with diabetes have excess mortality risk compared with those without diabetes after adjusting for competing causes of death. Beyond that, an independent effect of diabetes on mortality was found in women with mild, moderate and severe hyperglycaemia even after adjustment for cardiovascular risks and prevalent atherosclerotic diseases. It might be expected that severe hyperglycaemia would be associated with increased mortality, but mild/moderate hyperglycaemia is not always considered as important to health outcomes as cardiovascular comorbidities in older diabetics. Our finding of a trend of increasing mortality risk with increasing hyperglycaemia suggests that hyperglycaemia itself may, in part, contribute to excess mortality.

There are several biologically plausible pathways through which hyperglycaemia could affect mortality: through effects of hyperglycaemia on worsening vascular disease, direct vascular toxicity of hyperglycaemia, or through an association of hyperglycaemia with inflammatory or other biomediators. The effect of hyperglycaemia on mortality brings to the forefront the issue of glycaemic control in older, disabled people with diabetes. Although severe hyperglycaemia may be most problematic, mild and moderate also have an effect when compared with normoglycaemia.

Diabetes with increased hyperglycaemia may confer increased risk of mortality because increasingly severe hyperglycaemia can be viewed as a marker for severe, longstanding diabetes. It is clear from the UKPDS that the progression of Type 2 diabetes is associated with the progression of hyperglycaemia [31]. Severe hyperglycaemia is associated with increased diabetes duration, increased management difficulty, and increased adverse outcomes, particularly microvascular complications [32]. However, whether the mortality gradient associated with increasing levels of hyperglycaemia is considered a dose-response and/or a diabetes severity effect, this excess mortality is not completely explained by atherosclerotic complications of diabetes.

Although not the focus of this paper, our models also demonstrate other clinical covariates associated with excess mortality. Most are expected, such as heart failure and coronary artery disease. Low BMI and low cholesterol are associated with mortality in this sample of older disabled women. This is consistent with previous research that has also shown both low BMI $[23,33,34]$ and low cholesterol [35-37] to be associated with excess mortality in older adults, probably because they are markers for disease and/or undernutrition. The relationship of BMI to mortality in older adults has been noted to be 'U-shaped', with both low and high BMI associated with increased mortality [33]. Unfortunately, these observational associations still do not answer questions about the risk and benefits of weight loss among older adults, particularly those with chronic diseases. Low cholesterol in some older men may be related to both decreased synthesis and absorption [38] and has been associated with low albumin [35,37]. However, evidence that older adults with atherosclerotic disease benefit from cholesterol lowering is accumulating, and differences in pathophysiology of different chronic diseases will probably be shown to explain apparent differences in cholesterol and health outcomes in older people. In our sample of older women studied during the early 1990s, cholesterol-lowering medications would rarely have been prescribed, but that information was not available for this study.

It appears from these data that the effect of diabetes on mortality in older disabled women occurs via a broader range of pathways than clinical vascular disease alone. In our population-based study, diabetes at either level of hyperglycaemia showed an independent HR for all-cause mortality after statistically controlling for vascular disease risks and presence of vascular disease. In addition, there appears to be increased HR of non-cardiovascular mortality due to diabetes with either mild/moderate or severe hyperglycaemia, although the number of deaths among women with diabetes was small. Other studies have also found an increased risk for non-cardiovascular mortality in people with diabetes $[1,3,10,14,39,40]$. In our study, the absence of cardiovascular mortality risk may be a type 2 error due to relatively small sample size or related to self-exclusion of women with long-term consequences attributable to diabetes and hyperglycaemia. However, it is clinically plausible that for older disabled women, who have survived for many years with their diabetes and prevalent vascular disease, diabetes is associated with general debility leading to death from non-cardiovascular causes. Further study is needed to confirm and better understand this increased risk of noncardiovascular death in this subpopulation of older adults.

A limitation of our study was the relatively small number of women with diabetes. We were able to include women without previously diagnosed diabetes due to the inclusion of other clinical data to support this diagnosis. It is likely that women remain in the non-diabetic group who would be diagnosed with diabetes under current criteria. However, this misclassification bias should have caused attenuation of our findings so that a greater difference between the non-diabetic women and those with mild and moderate hyperglycaemia would be expected with improved classification. In addition, because of the lack of standardization among older GHB assays, the actual value of GHB in our study is not meaningful. However, categorization is a reasonable way to test the dose-response hypothesis regarding the effect of severe hyperglycaemia on mortality. Another limitation is that neither preclinical atherosclerotic disease nor severity of vascular disease was measured in our study, leading to possible incomplete adjustment for vascular disease. Finally, variables were measured at baseline, so incident and worsening vascular disease could not be included in our models, although we did study cardiovascular deaths.

Despite these limitations, our study had the benefit of a cohort of disabled women living in the community, a group who ordinarily exclude themselves or are excluded from 
research studies. The use of sampling from the Medicare database instead of volunteer recruitment, and home visits instead of travel to a study clinic, allowed us to study a representative subset of community-dwelling disabled women. We had extensive covariate data, including adjudicated chronic diseases, GHB, cholesterol, BMI, mortality and cause of death ascertainment. The significance of our findings despite low statistical power supports the potential importance of these observations, which heighten questions about the importance of hyperglycaemia to mortality in older disabled women. This population is of great clinical importance as a rapidly growing demographic group in the USA with a nearly $20 \%$ prevalence of diabetes [41]. These older diabetic women have all the characteristics that confuse treatment approaches toward diabetes in older adults: long diabetes duration; high prevalence of vascular complications; high prevalence of other comorbid conditions related and unrelated to diabetes; high prevalence of disability; and selective survival, by definition. Our data demonstrate an effect of hyperglycaemia on all-cause mortality over 6 years in disabled older women that is not fully explained by vascular disease; this effect increases with increasing hyperglycaemia. These findings suggest the need to manage moderate and severe hyperglycaemia in even the oldest, most disabled women in the community; more research is needed to understand the risks and benefits of managing mild hyperglycaemia in older women.

\section{Competing interests}

This research was supported by the U.S. HHS PHS National Institutes of Health grants (K08 AG0074901) to C.S.B. The WHAS I study was supported by contract (N01 AG12112) from the National Institute on Aging.

\section{Acknowledgements}

The authors thank Tisha Moore for her manuscript preparation and assistance with development of figures and tables.

\section{References}

1 Roper N, Bilous R, Kelly W, Unwin N, Connolly V. Cause-specific mortality in a population with diabetes: South Tees Diabetes Mortality Study. Diabetes Care 2002; 25: 43-48.

2 Bertoni A, Krop J, Anderson G, Brancati F. Diabetes-related morbidity and mortality in a national sample of U.S. elders. Diabetes Care 2002; 25: 471-475.

3 Tierney EF, Geiss LS, Engelgau MM, Thompson TJ, Schaubert D, Shireley LA et al. Population-based estimates of mortality associated with diabetes: use of a death certificate check box in North Dakota. Am J Public Health 2001; 91: 84-92.

4 Rockwood K, Awalt E, MacKnight C, McDowell I. Incidence and outcomes of diabetes mellitus in elderly people: report from the Canadian Study of Health and Aging. Can Med Assoc J 2000; 162: 769-772.

5 Berger B, Stenstrom G, Sundkvist G. Incidence, prevalence, and mortality of diabetes in a large population. A report from the Skaraborg Diabetes Registry. Diabetes Care 1999; 22: 773-778.
6 Vilbergsson S, Sigurdsson G, Sigvaldason H, Sigfusson N. Coronary heart disease mortality amongst non-insulin-dependent diabetic subjects in Iceland: the independent effect of diabetes. The Reykjavik Study 17-year follow up. J Intern Med 1998; 244: 309-316.

7 Muggeo M, Verlato G, Bonora E, Bressan F, Girotto S, Corbellini M et al. The Verona diabetes study: a population-based survey on known diabetes mellitus prevalence and 5 -year all-cause mortality. Diabetologia 1995; 38: 318-325.

8 Walters DP, Gatling W, Houston AC, Mullee MA, Julious SA, Hill RD. Mortality in diabetic subjects: an eleven-year follow-up of a community-based population. Diabet Med 1994; 11: 968-973.

9 Croxson SC, Price DE, Burden M, Jagger C, Burden AC. The mortality of elderly people with diabetes. Diabet Med 1994; 11: 250-252.

10 Stengard JH, Tuomilehto J, Pekkanen J, Kivinen P, Kaarsalo E, Nissinen A et al. Diabetes mellitus, impaired glucose tolerance and mortality among elderly men: the Finnish cohorts of the Seven Countries Study. Diabetologia 1992; 35: 760-765.

11 Knuiman MW, Welborn TA, Whittall DE. An analysis of excess mortality rates for persons with non-insulin-dependent diabetes mellitus in Western Australia using the Cox proportional hazards regression model. Am J Epidemiol 1992; 135: 638-648.

12 Waugh NR, Dallas JH, Jung RT, Newton RW. Mortality in a cohort of diabetic patients. Causes and relative risks. Diabetologia 1989; 32: 103-104.

13 Panzram G, Zabel-Langhennig R. Prognosis of diabetes mellitus in a geographically defined population. Diabetologia 1981; 20:587-591.

14 Khaw KT, Wareham N, Luben R, Bingham S, Oakes W, Welch A et al. Glycated haemoglobin, diabetes and mortality in men in Norfolk cohort of European prospective investigation of cancer and nutrition (EPIC-Norfolk). Br Med J 2001; 322: 15-28.

15 Guralnik J, Fried L, Simonsick E, Kasper J, Lafferty M. The Women's Health and Aging Study: Health and Social Charactersitics of Older Women with Disability. NIH Pub. no. 95-4009. Bethesda, MD: National Institute on Aging, 1995.

16 Fried LP, Ettinger WH, Lind B, Newman AB, Gardin J. Physical disability in older adults: a physiological approach. Cardiovascular Health Study Research Group. J Clin Epidemiol 1994; 47: 747-760.

17 Folstein MF, Folstein SE, McHugh PR. 'Mini-mental State'. A practical method of grading the cognitive status of patients for the clinician. J Psychiatr Res 1975; 12: 189-198.

18 Simonsick EM, Maffeo CE, Rogers SK, Skinner EA, Davis D, Guralnik JM et al. Methodology and feasibility of a home-based examination in disabled older women: the Women's Health and Aging Study. J Gerontol A Biol Sci Med Sci 1997; 52: M264-274.

19 Fried LP, Kasper J, Williamson JD, Skinner EA, Morris C, Hochberg M. Disease ascertainment algorithms. In Guralnik J, Fried LP, Simonsick EM, Kasper J, Lafferty M eds. The Women's Health and Aging Study: Health and Social Characteristics of Older Women with Disability. Appendix E. Bethesda, MD: National Institute on Aging, 1995.

20 Volpato S, Blaum CS, Resnick HE, Ferrucci L, Fried LP, Guralnik J. Comorbidities and impairments explaining the association between diabetes mellitus and lower extremity disability. The Women's Health and Aging Study. Diabetes Care 2002; 25: 678-683.

21 Newman AB, Siscovick DS, Manolio TA, Polak J, Fried LP, Borhani NO et al. Ankle-arm index as a marker of atherosclerosis in the Cardiovascular Health Study. Cardiovascular Heart Study (CHS) Collaborative Research Group. Circulation 1993; 88: 837-845.

22 Simonsick EM, Fried LP. Exercise tolerance and body composition. In Guralnik JM, Fried LP, Simonsick E, Kasper J, Lafferty M eds. The Women's Health and Aging Study: Health and Social Characteristics of Older Women with Disability. NIH Pub. No. 95-4009. Bethesda, MD: NIH, 1995; 106-117.

23 Fried LP, Kronmal RA, Newman AB, Bild DE, Mittelmark MB, Polak JF et al. Risk factors for 5 -year mortality in older adults: the Cardiovascular Health Study. JAMA 1998; 279: 585-592. 
24 Yesavage JA, Brink TL, Rose TL, Lum O, Huang V, Adey M et al. Development and validation of a geriatric depression screening scale: a preliminary report. J Psychiatr Res 1982; 17: 37-49.

25 Lyness JM, Noel TK, Cox C, King DA, Conwell Y, Caine ED. Screening for depression in elderly primary care patients. A comparison of the Center for Epidemiologic Studies-Depression Scale and the Geriatric Depression Scale. Arch Intern Med 1997; 157: 449-454.

26 The Diabetes Control and Complications Trial Research Group. The effect of intensive treatment of diabetes on the development and progression of long-term complications in insulin-dependent diabetes mellitus. N Engl J Med 1993; 329: 1676-1685.

27 Volpato S, Guralnik J, Ferrucci L, Balfour J, Chaves P, Fried LP et al. Cardiovascular disease, interleukin-6, and risk of mortality in women: The Women's Health and Aging Study. Circulation 2001; 103: 947-953.

28 Agresti A. Categorical Data Analysis. New York: John Wiley and Sons, 1990.

29 SAS Institute. SAS Procedures Guide, Version 8, Vols 1 and 2. Cary, NC: SAS Institute, 2000.

30 Stata Corporation. Stata User's Guide. College Station, TX: Stata Press, 2001.

31 UK Prospective Diabetes Study Group. Intensive blood-glucose control with sulphonylureas or insulin compared with conventional treatment and risk of complications in patients with type 2 diabetes. Lancet 1998; 352: 837-852.

32 Klein R. Hyperglycemia and microvascular and macrovascular disease in diabetes. Diabetes Care 1995; 18: 258-268.

33 Allison DB, Gallagher D, Heo M, Pi-Sunyer FX, Heymsfield SB. Body mass index and all-cause mortality among people age 70 and over: the Longitudinal Study of Aging. Int J Obes Relat Metab Disord 1997; 21: 424-431.
34 Landi F, Zuccala G, Gambassi G, Incalzi RA, Manigrasso L, Pagano $\mathrm{F}$ et al. Body mass index and mortality among older people living in the community. J Am Geriatr Soc 1999; 47: 1072-1076.

35 Volpato S, Leveille SG, Corti MC, Harris TB, Guralnik JM. The value of serum albumin and high-density lipoprotein cholesterol in defining mortality risk in older persons with low serum cholesterol. $J$ Am Geriatr Soc 2001; 49: 1142-1147.

36 Schatz IJ, Masaki K, Yano K, Chen R, Rodriguez BL, Curb JD. Cholesterol and all-cause mortality in elderly people from the Honolulu Heart Program: a cohort study. Lancet 2001; 358: 351355.

37 Reuben DB, Ix JH, Greendale GA, Seeman TE. The predictive value of combined hypoalbuminemia and hypocholesterolemia in high functioning community-dwelling older persons. MacArthur Studies of Successful Aging. J Am Geriatr Soc 1999; 47: 402-406.

38 Gylling H, Strandberg T, Tilvis R, Miettinen TA. Regulation of serum cholesterol level in middle-aged and elderly men. Relation of cholesterol absorption and synthesis to lipoprotein metabolism. Arterioscler Thromb 1994; 14: 694-700.

$39 \mathrm{Gu} \mathrm{K}$, Cowie CC, Harris MI. Mortality in adults with and without diabetes in a national cohort of the U.S. population, 1971-1993. Diabetes Care 1998; 21: 1138-1145.

40 Koskinen SV, Martelin TP, Valkonen T. Socioeconomic differences in mortality among diabetic people in Finland: five year follow up. Br Med J 1996; 313: 975-978.

41 Harris MI, Flegal KM, Cowie CC, Eberhardt MS, Goldstein DE, Little RR et al. Prevalence of diabetes, impaired fasting glucose, and impaired glucose tolerance in U.S. adults. The Third National Health and Nutrition Examination Survey, 1988-1994. Diabetes Care 1998; 21: 518-524. 